\title{
Projective Metrizability and Formal Integrability
}

\author{
Ioan BUCATARU ${ }^{\dagger}$ and Zoltán MUZSNAY ${ }^{\ddagger}$ \\ $\dagger$ Faculty of Mathematics, Al.I.Cuza University, B-dul Carol 11, Iasi, 700506, Romania \\ E-mail: bucataru@uaic.ro \\ URL: http://www.math.uaic.ro/ bucataru/ \\ $\ddagger$ Institute of Mathematics, University of Debrecen, H-4010 Debrecen, Pf. 12, Hungary \\ E-mail: muzsnay@science.unideb.hu \\ URL: http://www.math.klte.hu/ muzsnay/
}

Received August 25, 2011, in final form December 08, 2011; Published online December 12, 2011

http://dx.doi.org/10.3842/SIGMA.2011.114

\begin{abstract}
The projective metrizability problem can be formulated as follows: under what conditions the geodesics of a given spray coincide with the geodesics of some Finsler space, as oriented curves. In Theorem 3.8 we reformulate the projective metrizability problem for a spray in terms of a first-order partial differential operator $P_{1}$ and a set of algebraic conditions on semi-basic 1-forms. We discuss the formal integrability of $P_{1}$ using two sufficient conditions provided by Cartan-Kähler theorem. We prove in Theorem 4.2 that the symbol of $P_{1}$ is involutive and hence one of the two conditions is always satisfied. While discussing the second condition, in Theorem 4.3 we prove that there is only one obstruction to the formal integrability of $P_{1}$, and this obstruction is due to the curvature tensor of the induced nonlinear connection. When the curvature obstruction is satisfied, the projective metrizability problem reduces to the discussion of the algebraic conditions, which as we show are always satisfied in the analytic case. Based on these results, we recover all classes of sprays that are known to be projectively metrizable: flat sprays, isotropic sprays, and arbitrary sprays on 1- and 2-dimensional manifolds. We provide examples of sprays that are projectively metrizable without being Finsler metrizable.
\end{abstract}

Key words: sprays; projective metrizability; semi-basic forms; partial differential operators; formal integrability

2010 Mathematics Subject Classification: 49N45; 58E30; 53C60; 58B20; 53C22

\section{Introduction}

The projective metrizability problem for a homogeneous system of second-order ordinary differential equations, which can be identified with a spray $S$, seeks for a Finsler metric $F$ whose geodesics coincide with the geodesics of the spray $S$, up to an orientation preserving reparameterization. For the case when $S$ is a flat spray this problem was first studied by Hamel [16] and it is known as the Finslerian version of Hilbert's fourth problem $[1,11,30]$. In the general case it was Rapcsák [27] who obtained, in local coordinates, necessary and sufficient conditions for the projective metrizability problem of a spray. Global formulations for the projective metrizability problem where obtained by Klein and Voutier [17], and by Szilasi and Vattamány [31]. It has been shown that this is an essential problem in various fields of biology and physics [3].

The projective metrizability problem can be formulated as a particular case of the inverse problem of the calculus of variations. We refer to $[2,8,19,25,28]$ for various approaches of the inverse problem of the calculus of variations. One of this approaches seeks for the existence of a multiplier matrix that satisfies four Helmholtz conditions [19, 28]. In [5], these four Helmholtz conditions where reformulated in terms of a semi-basic 1-form. For the particular 
case of the projective metrizability problem, it has been shown in [5] that only two of the four Helmholtz conditions are independent. In this work we discuss the formal integrability of these two Helmholtz conditions using two sufficient conditions provided by Cartan-Kähler theorem. The approach in this work follows the one developed in [26] for studying the Finsler metrizability problem for a spray.

In Section 2 we recall first some basic aspects of the Frölicher-Nijenhuis theory on a manifold $M[13,18]$. Then, we use this theory on $T M$ and apply it to the natural objects that live on the tangent space: vertical distribution, Liouville vector field, and semi-basic forms $[14,15,20]$.

In Section 3 we use the geometric setting developed in the previous section to reformulate the projective metrizability problem. In Theorem 3.8 we obtain a set of necessary and sufficient conditions, for the projective metrizability problem of a spray, which consists of a set of algebraic equations (3.7) and a set of differential equations (3.8) on semi-basic forms. The set of differential equations determine a first-order partial differential operator $P_{1}$, called the projective metrizability operator, which acts on semi-basic 1-forms.

In Section 4 we discuss the formal integrability of the projective metrizability operator $P_{1}$, using two sufficient conditions provided by Cartan-Kähler theorem. Based on this theorem and Theorems 4.2 and 4.3 we conclude that there is only one obstruction to the formal integrability of $P_{1}$. This obstruction is expressed in terms of the curvature tensor of the nonlinear connection induced by the spray. In this work we pay attention to various cases when the obstruction condition is automatically satisfied. Another possibility, which we leave for further work, is to add this obstruction to the projective metrizability operator and discuss the formal integrability of the new operator. Using different techniques, an alternative expression of the obstruction condition was obtain in [31, Theorem 4.9].

In Section 5 we discuss some classes of sprays for which the curvature obstruction is automatically satisfied: flat sprays, isotropic sprays, and arbitrary sprays on 2-dimensional manifolds. For each of these classes of sprays, the projective metrizability problem reduces to the discussion of the algebraic conditions (3.7), which as we show are always satisfied in the analytic case. Although, for these classes, the projective metrizability problem has been discussed before by some authors, our approach in this work is different. Using different methods, it was demonstrated in [9] that flat sprays are projectively metrizable. In [10] it has been shown that isotropic sprays are projectively equivalent with flat sprays, and hence are projectively metrizable. On a 2dimensional manifold it has been shown by Matsumoto that every spray is projectively related to a Finsler space [22], by extending the original discussion of Darboux [12] about second-order differential equations.

We use a spray on a 2-dimensional, considered by Anderson and Thompson in [2], and a projectively flat spray of constant flag curvature, considered by Yang in [32], to provide examples of projectively metrizable sprays that are not Finsler metrizable.

\section{Preliminaries}

In this section we present the differential geometric tools we need to formulate and study the projective metrizability problem.

A systems of second-order ordinary differential equations on a manifold $M$ can be identified with a second-order vector field that is called a semispray. To each semispray one can associate a geometric apparatus very useful to obtain qualitative information regarding: the variations of its geodesics, their stability, as well as the inverse problem of the calculus of variations, [4]. A global formulation for the geometric apparatus one can associate to a semispray is due to Grifone [14] and can be obtained using the Frölicher-Nijenhuis theory [13]. 


\subsection{Frölicher-Nijenhuis theory}

In this subsection we recall and extend some aspects of the Frölicher-Nijenhuis theory, which will be applied in the next subsection to vector valued differential forms on tangent bundles. For the classic and modern formulations of Frölicher-Nijenhuis theory we refer to $[13,14,15,18,20]$.

In this work $M$ is a real, $n$-dimensional, smooth manifold. We denote by $C^{\infty}(M)$, the ring of smooth functions on $M$, and by $\mathfrak{X}(M)$, the $C^{\infty}(M)$-module of vector fields on $M$. Consider $\Lambda(M)=\bigoplus_{k \in \mathbb{N}} \Lambda^{k}(M)$ the graded algebra of differential forms on $M$. We denote by $S^{k}(M)$ the space of symmetric $(0, k)$ tensors on $M$. We also write $\Psi(M)=\bigoplus_{k \in \mathbb{N}} \Psi^{k}(M)$ for the graded algebra of vector-valued differential forms on $M$.

For $L \in \Psi^{l}(M)$, a vector valued $l$-form, we consider $\tau_{L}: \Lambda^{1}(M) \otimes \Lambda^{k}(M) \rightarrow \Lambda^{k+l}(M)$, or $\tau_{L}: \Psi^{1}(M) \otimes \Psi^{k}(M) \rightarrow \Psi^{k+l}(M)$, the alternating operator defined as

$$
\left(\tau_{L} B\right)\left(X_{1}, \ldots, X_{k+l}\right)=\frac{1}{k ! l !} \sum_{\sigma \in S_{k+l}} \varepsilon(\sigma) B\left(L\left(X_{\sigma(1)}, \ldots, X_{\sigma(l)}\right), X_{\sigma(l+1)}, \ldots, X_{\sigma(l+k)}\right),
$$

where $X_{1}, \ldots, X_{k+l} \in \mathfrak{X}(M)$ and $S_{k+l}$ is the permutation group of $\{1, \ldots, k+l\}$.

The restriction of $\tau_{L}$ to $\Lambda^{k+1}(M) \subset \Lambda^{1}(M) \otimes \Lambda^{k}(M)$, or the restriction to $\Psi^{k+1}(M)$, is a derivation of degree $(l-1)$ and it coincides with the inner product $i_{L}$, see $[15,18]$. Inner product $i_{L}$ is trivial on $\Lambda^{0}(M)=C^{\infty}(M)$, or $\Psi^{0}(M)=\mathfrak{X}(M)$, and hence it is a derivation of type $i_{*}$ [15] or an algebraic derivation [18]. Since it satisfies the Leibniz rule, $i_{L}$ is uniquely determined by its action on $\Lambda^{1}(M)$, or $\Psi^{1}(M)$, when it is given by $i_{L} B=B \circ L$. For the particular case when $l=1$ and $L=\mathrm{Id}$ we have that $i_{\mathrm{Id}} B=k B$ for all $B \in \Lambda^{k}(M)$, or $B \in \Psi^{k}(M)$.

For a linear connection $\nabla$ on $M$ consider $d^{\nabla}: \Psi^{k}(M) \rightarrow \Psi^{k+1}(M)$ the covariant exterior derivative, see $[18, \S 11.13]$, given by

$$
\begin{aligned}
d^{\nabla} B\left(X_{1}, \ldots, X_{k+1}\right)= & \sum_{i=1}^{k+1}(-1)^{i+1} \nabla_{X_{i}} B\left(X_{1}, \ldots, \hat{X}_{i}, \ldots, X_{l+1}\right) \\
& +\sum_{1 \leq i<j \leq k+1}(-1)^{i+j} B\left(\left[X_{i}, X_{j}\right], X_{1}, \ldots, \hat{X}_{i}, \ldots, \hat{X}_{j}, \ldots, X_{k+1}\right) .
\end{aligned}
$$

The exterior derivative $d: \Lambda^{k}(M) \rightarrow \Lambda^{k+1}(M)$ satisfies also formula (2.2) for $B \in \Lambda^{k}(M)$. Therefore, we will use the notation $d^{\nabla}$ to refer to both, the covariant exterior derivative, or the exterior derivative. For the latter case $d=d^{\nabla}$ does not depend on the linear connection $\nabla$.

For a vector valued $l$-form $L$, consider the commutator of the inner product $i_{L}$ and the (covariant) exterior derivative $d\left(d^{\nabla}\right)$. This differential operator is denoted by $d_{L}: \Lambda^{k}(M) \rightarrow$ $\Lambda^{k+l}(M)\left(d_{L}^{\nabla}: \Psi^{k}(M) \rightarrow \Psi^{k+l}(M)\right)$, it is given by

$$
d_{L}^{\nabla}=i_{L} \circ d^{\nabla}+(-1)^{l} d^{\nabla} \circ i_{L},
$$

it is a derivation of degree $l$, which is called the (covariant) exterior derivative with respect to $L$. Derivation $d_{L}\left(d_{L}^{\nabla}\right)$ commutes with the exterior derivative $d\left(d^{\nabla}\right)$ and hence it is a derivation of type $d_{*}[15]$ or a Lie derivation [18]. Since it satisfies the Leibniz rule, $d_{L}\left(d_{L}^{\nabla}\right)$ is uniquely determined by its action on $\Lambda^{0}(M)=C^{\infty}(M)\left(\Psi^{0}(M)=\mathfrak{X}(M)\right)$. For the particular case when $l=1$ and $L=$ Id we have that $d_{\mathrm{Id}}^{\nabla}=d^{\nabla}$. Therefore, we obtain $d^{\nabla} \mathrm{Id}=T$, where $T$ is the torsion of the linear connection $\nabla$.

For two vector valued forms $L \in \Psi^{l}(M)$ and $K \in \Psi^{k}(M)$, their Frölicher-Nijenhuis bracket $[L, K]$ is a vector valued $(k+l)$-form, defined by

$$
d_{[L, K]}=d_{L} \circ d_{K}-(-1)^{k l} d_{K} \circ d_{L}
$$


For a vector valued $l$-form $L$ and a linear connection $\nabla$ on $M$ we obtain a derivation of degree $l$ given by $\mathcal{D}_{L}=\tau_{L} \nabla$. Hence, $\mathcal{D}_{L}: \Lambda^{k}(M) \rightarrow \Lambda^{k+l}(M)\left(\mathcal{D}_{L}: \Psi^{k}(M) \rightarrow \Psi^{k+l}(M)\right)$, acts on (vector-valued) $k$-forms as follows:

$$
\left(\mathcal{D}_{L} B\right)\left(X_{1}, \ldots, X_{k+l}\right)=\frac{1}{l ! k !} \sum_{\sigma \in S_{k+l}} \varepsilon(\sigma)\left(\nabla_{L\left(X_{\sigma(1)}, \ldots, X_{\sigma(l)}\right)} B\right)\left(X_{\sigma(l+1)}, \ldots, X_{\sigma(k+l)}\right) .
$$

For the particular case when $l=1$ and $L=\mathrm{Id}$, we denote the corresponding derivation of degree 1 by $\mathcal{D}=\mathcal{D}_{\text {Id }}$. Since any derivation of degree $l$ can be uniquely decomposed into a sum of a Lie derivation and an algebraic derivation [18, $\S 8.3$, we obtain for $\mathcal{D}_{L}$ the following result.

Lemma 2.1. For a vector valued $l$-form $L$ and a linear connection $\nabla$ on $M$, derivation of degree $l, \mathcal{D}_{L}$, decomposes uniquely into a sum of a Lie derivation and an algebraic derivation as follows:

$$
\mathcal{D}_{L}=d_{L}^{\nabla}-i_{d_{L}^{\nabla} \mathrm{Id}}
$$

Proof. When acting on forms, formula (2.6) reads $\mathcal{D}_{L}=d_{L}-i_{d_{L}^{\nabla} \mathrm{Id}}$. The vector valued $(l+1)$ form that defines the inner product in formula (2.6) is, according to formula (2.3), given by $d_{L}^{\nabla} \mathrm{Id}=i_{L} T+(-1)^{l} d^{\nabla} L$.

Since Lie derivations commute with the exterior derivative $d^{\nabla}$ and satisfy the Leibnitz rule it follows that they are uniquely determined by their action on $\Lambda^{0}(M)=C^{\infty}(M)\left(\Psi^{0}(M)=\right.$ $\mathfrak{X}(M)$ ). Using formulae (2.3) and (2.5) one can immediately check that $\mathcal{D}_{L} f=d_{L} f$ for any scalar (vector valued) 0 -form $f$.

Since algebraic derivations are trivial on $\Lambda^{0}(M)=C^{\infty}(M)\left(\Psi^{0}(M)=\mathfrak{X}(M)\right)$, and satisfy the Leibnitz rule it follows that they are uniquely determined by their action on $\Lambda^{1}(M)\left(\Psi^{1}(M)\right)$. To prove formula (2.6) we have to show that

$$
\left(\mathcal{D}_{L}-d_{L}^{\nabla}\right) \omega=-\omega \circ\left(d_{L}^{\nabla} \mathrm{Id}\right)
$$

for any (vector valued) 1-form $\omega$. Since formally, we have the same formulae to define the action on scalar, or vector valued forms, we will work with scalar forms.

Let $\omega \in \Lambda^{1}(M)$ and $X_{1}, \ldots, X_{l+1} \in \mathfrak{X}(M)$. For $k=1$, from formula (2.5) we obtain the action of the derivation $\mathcal{D}_{L}$ on 1 -forms as follows:

$$
\begin{aligned}
\left(\mathcal{D}_{L} \omega\right) & \left(X_{1}, \ldots, X_{l+1}\right)=\sum_{i=1}^{l+1}(-1)^{l+1-i}\left(\nabla_{L\left(X_{1}, \ldots, \hat{X}_{i}, \ldots, X_{l+1}\right)} \omega\right)\left(X_{i}\right) \\
= & \sum_{i=1}^{l+1}(-1)^{l+1-i}\left\{L\left(X_{1}, \ldots, \hat{X}_{i}, \ldots, X_{l+1}\right)\left(\omega\left(X_{i}\right)\right)-\omega\left(\nabla_{L\left(X_{1}, \ldots, \hat{X}_{i}, \ldots, X_{l+1}\right)} X_{i}\right)\right\} .
\end{aligned}
$$

From formula (2.3) we obtain that the action of the exterior derivative $d_{L}$ on a 1 -form $\omega$ is given by $d_{L} \omega=i_{L} d \omega+(-1)^{l} d(\omega \circ L)$. Therefore, for $X_{1}, \ldots, X_{l+1} \in \mathfrak{X}(M)$ we have

$$
\begin{aligned}
\left(d_{L} \omega\right)\left(X_{1}, \ldots, X_{l+1}\right)= & \sum_{i=1}^{l+1}(-1)^{l+1-i} L\left(X_{1}, \ldots, \hat{X}_{i}, \ldots, X_{l+1}\right)\left(\omega\left(X_{i}\right)\right) \\
& +\sum_{i=1}^{l+1}(-1)^{l+1-i} \omega\left(\left[X_{i}, L\left(X_{1}, \ldots, \hat{X}_{i}, \ldots, X_{l+1}\right)\right]\right) \\
& +\sum_{1 \leq i<j \leq l+1}(-1)^{l+i+j} \omega\left(L\left(\left[X_{i}, X_{j}\right], X_{1}, \ldots, \hat{X}_{i}, \ldots, \hat{X}_{j}, \ldots, X_{l+1}\right)\right) .
\end{aligned}
$$

Now, we evaluate $d_{L}^{\nabla} \mathrm{Id}=i_{L} T+(-1)^{l} d^{\nabla} L$ on $l+1$ vectors $X_{1}, \ldots, X_{l+1} \in \mathfrak{X}(M)$. 
For $k=1$, if we restrict the action of $\tau_{L}$ given by formula $(2.1)$ to $\Psi^{2}(M)$ we obtain that the inner product $i_{L}: \Psi^{2}(M) \rightarrow \Psi^{l+1}(M)$ is given by:

$$
\left(i_{L} T\right)\left(X_{1}, \ldots, X_{l+1}\right)=\sum_{i=1}^{l+1}(-1)^{l-i} T\left(X_{i}, L\left(X_{1}, \ldots, \hat{X}_{i}, \ldots, X_{l+1}\right)\right) .
$$

Using formula (2.2), the action of the exterior covariant derivative $d^{\nabla}$ on the vector valued $l$-form $L$ is given by

$$
\begin{aligned}
d^{\nabla} L\left(X_{1}, \ldots, X_{l+1}\right)= & \sum_{i=1}^{l+1}(-1)^{i+1} \nabla_{X_{i}} L\left(X_{1}, \ldots, \hat{X}_{i}, \ldots, X_{l+1}\right) \\
& +\sum_{1 \leq i<j \leq l+1}(-1)^{i+j} L\left(\left[X_{i}, X_{j}\right], X_{1}, \ldots, \hat{X}_{i}, \ldots, \hat{X}_{j}, \ldots, X_{l+1}\right) .
\end{aligned}
$$

Using formulae (2.7), (2.8), (2.9), and (2.10) it follows that

$$
\left(\mathcal{D}_{L} \omega-d_{L} \omega\right)\left(X_{1}, \ldots, X_{l+1}\right)=-\left(\omega \circ\left(i_{L} T+(-1)^{l} d^{\nabla} L\right)\right)\left(X_{1}, \ldots, X_{l+1}\right),
$$

for all $X_{1}, \ldots, X_{l+1} \in \mathfrak{X}(M)$, which means that the decomposition (2.6) is true.

For the particular case when $l=1$ and $L=\mathrm{Id}$, we have that the vector valued 2-form $d_{\mathrm{Id}}^{\nabla} \mathrm{Id}$ reduces to torsion $T$ since $i_{\mathrm{Id}} T=2 T, d^{\nabla} \mathrm{Id}=T$. Therefore, decomposition (2.6) becomes

$$
\mathcal{D}=d-i_{T}
$$

Remark 2.2. Formula (2.6) shows that the difference of the two derivations $d_{L}-\mathcal{D}_{L}=i_{d_{L}^{\nabla} \mathrm{Id}}$ is an algebraic derivation. In other words, if $\omega \in \Lambda^{k}(M)$ vanishes at some point $p \in M, \omega_{p}=0$, then $\left(\mathcal{D}_{L} \omega\right)_{p}=\left(d_{L} \omega\right)_{p}$. For the particular case when $l=1$, this result has been shown in [15, Proposition 2.5].

\subsection{Vertical calculus on $T M$ and semi-basic forms}

Consider $(T M, \pi, M)$, the tangent bundle of the manifold $M$ and $(T M \backslash\{0\}), \pi, M)$ the slashed tangent bundle, which is the tangent bundle with the zero section removed. The tangent bundle carries some canonical structures, such as the vertical distribution, the Liouville vector field, and the vertical endomorphism. The differential calculus associated to these structures, using the Frölicher-Nijenhuis theory developed in the previous subsection, plays an important role in the geometry of a system of second-order ordinary differential equations, [4, 5, 14, 15, 17, 20, 24].

The vertical subbundle is defined as $V T M=\{\xi \in T T M,(D \pi)(\xi)=0\}$. It induces a vertical distribution $V: u \in T M \mapsto V_{u}=V T M \cap T_{u} T M$. This distribution is $n$-dimensional and it is integrable, being tangent to the leaves of the natural foliation induced by submersion $\pi$. If $\left(x^{i}\right)$ are local coordinates on the base manifold $M$, we denote by $\left(x^{i}, y^{i}\right)$ the induced coordinates on $T M$. It follows that $y^{i}$ are coordinates along the leaves of the natural foliation, while $x^{i}$ are transverse coordinates for the foliation. We denote by $\mathfrak{X}^{v}(T M)$ the Lie subalgebra of vertical vector fields on $T M$. An important vertical vector field on $T M$ is the Liouville vector field, which locally is given by $\mathbb{C}=y^{i} \partial / \partial y^{i}$.

The tangent structure (or vertical endomorphism) is the (1,1)-type tensor field $J$ on $T M$, which locally can be written as follows:

$$
J=\frac{\partial}{\partial y^{i}} \otimes d x^{i}
$$


Tensor $J$ satisfies $J^{2}=0$ and $\operatorname{Ker} J=\operatorname{Im} J=V T M$. Tangent structure $J$ is an integrable structure since the Frölicher-Nijenhuis bracket vanishes, $[J, J]=0$. As a consequence and using formula (2.4) we have that $d_{J}^{2}=0$.

For the natural foliation induced by submersion $\pi$ and the corresponding vertical distribution there are some important classes of forms: basic and semi-basic forms. As we will see in the next sections, semi-basic forms, vector valued semi-basic forms, and vector valued almost semi-basic forms are important ingredients to formulate and address the projective metrizability problem.

Definition 2.3. Consider $\omega \in \Lambda(T M)$ and $L \in \Psi(T M)$.

i) $\omega$ is called a basic form if both $\omega$ and $d \omega$ vanish whenever one of the arguments of $\omega$ (respectively $d \omega$ ) is a vertical vector field.

ii) $\omega$ is called a semi-basic form if it vanishes whenever one of its arguments is a vertical vector field.

iii) $L$ is called a vector valued semi-basic form if it takes vertical values and vanishes whenever one of its arguments is a vertical vector field.

iv) $L$ is called a vector valued almost semi-basic form if it vanishes whenever one of its arguments is a vertical vector field and for every vertical vector field $X \in \mathfrak{X}^{v}(T M)$ we have that $\mathcal{L}_{X} L$ is a vector valued semi-basic form.

In local coordinates a basic $k$-form $\omega$ on $T M$ can be written as

$$
\omega=\frac{1}{k !} \omega_{i_{1} \ldots i_{k}}(x) d x^{i_{1}} \wedge \cdots \wedge d x^{i_{k}} .
$$

For basic forms, the coordinates functions $\omega_{i_{1} \ldots i_{k}}(x)$ are basic functions, which means that they are constant along the leaves of the natural foliation.

Locally, a semi-basic $k$-form $\omega$ on $T M$ can be written as

$$
\omega=\frac{1}{k !} \omega_{i_{1} \ldots i_{k}}(x, y) d x^{i_{1}} \wedge \cdots \wedge d x^{i_{k}} .
$$

We will denote by $\Lambda_{v}^{k}(T M)$ the set of semi-basic $k$-forms on $T M$. A 1-form $\omega$ on $T M$ is semi-basic if and only if $i_{J} \omega=\omega \circ J=0$.

In local coordinates, a vector valued semi-basic $l$-form $L$ on $T M$ can be written as

$$
L=\frac{1}{l !} L_{i_{1} \ldots i_{l}}^{j}(x, y) \frac{\partial}{\partial y^{j}} \otimes d x^{i_{1}} \wedge \cdots \wedge d x^{i_{l}} .
$$

In this work all contravariant or covariant indices, of some tensorial coefficients, that refer to vertical components will be underlined. We will denote by $\Psi_{v}^{l}(T M)$ the set of vector valued semi-basic $l$-forms on $T M$. A vector valued 1-form $L$ on $T M$ is semi-basic if and only if $J \circ L=0$ and $i_{J} L=L \circ J=0$. The tangent structure $J$ is a vector valued semi-basic 1-form.

Locally, a vector valued almost semi-basic $l$-form $L$ on $T M$ can be expressed as

$$
L=\frac{1}{l !} L_{i_{1} \ldots i_{l}}^{j}(x) \frac{\partial}{\partial x^{j}} \otimes d x^{i_{1}} \wedge \cdots \wedge d x^{i_{l}}+\frac{1}{l !} L_{\bar{i}_{1} \ldots i_{l}}^{j}(x, y) \frac{\partial}{\partial y^{j}} \otimes d x^{i_{1}} \wedge \cdots \wedge d x^{i_{l}} .
$$

For a vector $X$ on $T M$ and a vector valued $l$-form $L$ on $T M$, the Frölicher-Nijenhuis bracket $[X, L]$, defined by formula (2.4), is a vector valued $l$-form on $T M$ given by:

$$
[X, L]\left(X_{1}, \ldots, X_{l}\right)=\left[X, L\left(X_{1}, \ldots, X_{l}\right)\right]-\sum_{i=1}^{l} L\left(\left[X, X_{i}\right], X_{1}, \ldots, \hat{X}_{i}, \ldots, X_{l}\right),
$$


for $X_{1}, \ldots, X_{l}$ vector fields on $T M$. Using the above formula and the fact that the vertical distribution is integrable it follows that vector valued semi-basic forms are also almost semibasic. This can be seen also from the local expressions (2.12) and (2.13).

Next two lemmas give a good motivation for considering the class of vector valued almost semi-basic forms. We will also see in Section 4 that the partial differential operator we use to discuss the projective metrizability problem is defined in terms of some vector valued almost semi-basic forms.

Lemma 2.4. Let $L$ be a vector valued almost semi-basic l-form on $T M$. Then, the differential operator $d_{L}$ preserves semi-basic forms, $d_{L}: \Lambda_{v}^{k}(T M) \rightarrow \Lambda_{v}^{k+l}(T M)$.

Proof. Consider a vector valued almost semi-basic $l$-form $L$, locally given by formula (2.13), and a semi-basic $k$-form $\omega$, locally given by formula (2.11). Since $d_{L}$ is a derivation of degree $l$, it follows that the $(k+l)$-form $d_{L} \omega$ can be expressed locally as follows

$$
\begin{aligned}
d_{L} \omega= & \frac{1}{k !} d_{L}\left(\omega_{i_{1} \cdots i_{k}}\right) \wedge d x^{i_{1}} \wedge \cdots \wedge d x^{i_{k}} \\
& +\frac{1}{k !} \sum_{j=1}^{k}(-1)^{(j-1) l} \omega_{i_{1} \cdots i_{k}} d x^{i_{1}} \wedge \cdots \wedge d_{L} d x^{i_{j}} \wedge \cdots \wedge d x^{i_{k}} .
\end{aligned}
$$

Using the assumption that $L$ is a vector valued almost semi-basic form, we show that all terms in the right hand side of the above formula are semi-basic forms. Since $L$ vanishes whenever one of its arguments is a vertical vector field it follows that for a function $f \in C^{\infty}(T M)$, $d_{L} f=i_{L} d f=d f \circ L$ is a semi-basic $l$-form. Hence $d_{L}\left(\omega_{i_{1} \cdots i_{k}}\right)$ are semi-basic $l$-forms.

We will prove now that $d_{L} d x^{i_{j}}=(-1)^{l} d d_{L} x^{i_{j}}$ are semi-basic $(l+1)$-forms. Using the local expression (2.13) of $L$, we have

$$
d_{L} x^{i_{j}}=i_{L} d x^{i_{j}}=d x^{i_{j}} \circ L=\frac{1}{l !} L_{i_{1} \ldots i_{l}}^{i_{j}}(x) d x^{i_{1}} \wedge \cdots \wedge d x^{i_{l}},
$$

which are basic $l$-forms. Therefore, $d d_{L} x^{i_{j}}$ are basic and hence semi-basic $(l+1)$-forms. One can conclude now that all terms in the right hand side of formula (2.14) are semi-basic forms and hence $d_{L} \omega$ is a semi-basic $(k+l)$-form.

Lemma 2.5. Consider $\nabla$ a linear connection on $T M$ such that $\nabla J=0$ and a vector valued almost semi-basic l-form $L$ on $T M$. Then, the differential operator $\mathcal{D}_{L}$ preserves semi-basic forms, $\mathcal{D}_{L}: \Lambda_{v}^{k}(T M) \rightarrow \Lambda_{v}^{k+l}(T M)$.

Proof. Using formula (2.6) and Lemma 2.4 we have that the differential operator $\mathcal{D}_{L}$ preserves semi-basic forms if and only if the algebraic derivation of degree $l, i_{d_{L}^{\nabla} \text { Id }}$ preserves semi-basic forms. Hence, we will complete the proof if we show that the vector valued $(l+1)$-form $d_{L}^{\nabla} \operatorname{Id}=$ $i_{L} T+(-1)^{l} d_{L}^{\nabla}$ takes vertical values whenever one of its arguments is a vertical vector field. Here $T$ is the torsion of the linear connection $\nabla$.

Using formulae (2.9) and (2.10) we have

$$
\begin{aligned}
(-1)^{l} & \left(d_{L}^{\nabla} \mathrm{Id}\right)\left(X_{1}, \ldots, X_{l+1}\right)=(-1)^{l}\left(i_{L} T+(-1)^{l} d_{L}^{\nabla}\right)\left(X_{1}, \ldots, X_{l+1}\right) \\
= & \sum_{i=1}^{l+1}(-1)^{i+1}\left\{\nabla_{L\left(X_{1}, \ldots, \hat{X}_{i}, \ldots, X_{l+1}\right)} X_{i}+\left[X_{i}, L\left(X_{1}, \ldots, \hat{X}_{i}, \ldots, X_{l+1}\right)\right]\right\} \\
& +\sum_{1 \leq i<j \leq l+1}(-1)^{i+j} L\left(\left[X_{i}, X_{j}\right], X_{1}, \ldots, \hat{X}_{i}, \ldots, \hat{X}_{j}, \ldots, X_{l+1}\right) .
\end{aligned}
$$

We will show now that whenever one of the $(l+1)$ arguments of $d_{L}^{\nabla}$ Id is a vertical vector field, then the right hand side of formula $(2.15)$ is a vertical vector field. Using the fact that $d_{L}^{\nabla} \mathrm{Id}$ 
is a vector valued $(l+1)$-form, we will discuss only the case when $X_{1}$ is a vertical vector field. Since $L$ vanishes whenever one of its arguments is a vertical vector field, the nonzero vector field that remains from the right hand side of formula (2.15), when $X_{1}$ is a vertical vector field, is

$$
\nabla_{L\left(X_{2}, \ldots, X_{l+1}\right)} X_{1}+\left[X_{1}, L\right]\left(X_{2}, \ldots, X_{l+1}\right)
$$

The condition $\nabla J=0$ implies that the linear connection $\nabla$ preserves the vertical distribution and since $X_{1}$ is a vertical vector field it follows that $\nabla_{L\left(X_{2}, \ldots, X_{l+1}\right)} X_{1}$ is a vertical vector field as well. Since $L$ is a vector valued almost semi-basic $l$-form and $X_{1}$ is a vertical vector field it follows that $\left[X_{1}, L\right]$ is a vector valued semi-basic form, therefore it takes values into the vertical distribution and hence $\left[X_{1}, L\right]\left(X_{2}, \ldots, X_{l+1}\right)$ is a vertical vector field. It follows that the vector field in formula (2.16) is vertical and hence we have completed the proof.

\section{Projective metrizability problem of a spray}

A system of homogeneous second-order ordinary differential equations on a manifold $M$, whose coefficients do not depend explicitly on time, can be identified with a special vector field on $T M$ that is called a spray. In this section we address the following question, known as the projective metrizability problem: for a given spray $S$ find necessary and sufficient conditions for the existence of a Finsler function $F$ such that the geodesics of $S$ and the geodesics of $F$ coincide up to an orientation preserving reparameterization. We obtain such necessary and sufficient conditions in Theorem 3.8 and these conditions are expressed in terms of semi-basic 1-forms.

Particular aspects of the projective metrizability problem were studied more than a century ago by Hamel [16]. The problem was formulated rigorously in 1960's by Rapcsák [27] and Klein and Voutier [17]. Yet, the projective metrizability problem is far from being solved, and in the last decade it has been intensively studied [1, 5, 10, 11, 29, 30, 31, 32].

\subsection{Spray, nonlinear connection, and curvature}

In this subsection, we start with a spray $S$ and use the Frölicher-Nijenhuis theory to derive a differential calculus on $T M \backslash\{0\}$ [14] and to obtain information about the given system of SODE. For the remaining part of the paper, all geometric objects will be considered defined on the slashed tangent bundle $T M \backslash\{0\}$ and not on the whole $T M$. This is motivated by the fact that we will want to connect them with geometric structures in Finsler geometry, where the Finsler function is not differentiable on the zero section.

Definition 3.1. A vector field $S \in \mathfrak{X}(T M \backslash\{0\})$ is called a spray if

i) $J S=\mathbb{C}$,

ii) $[\mathbb{C}, S]=S$.

First condition in Definition 3.1 expresses that a spray $S$ can be locally given as

$$
S=y^{i} \frac{\partial}{\partial x^{i}}-2 G^{i}(x, y) \frac{\partial}{\partial y^{i}}
$$

for some functions $G^{i}$ defined on domains of induced coordinates on $T M \backslash\{0\}$.

Second condition in Definition 3.1 expresses that the vector field $S$ is 2-homogeneous. It is equivalent with the fact that functions $G^{i}$ are 2-homogeneous in the fibre coordinates. In this work we will consider positive homogeneity only and hence $G^{i}(x, \lambda y)=\lambda^{2} G^{i}(x, y)$ for all $\lambda>0$. By Euler's theorem this homogeneity condition is equivalent to $\mathbb{C}\left(G^{i}\right)=2 G^{i}$. 
A curve $c: I \rightarrow M$ is called regular if its tangent lift takes values in the slashed tangent bundle, $c^{\prime}: I \rightarrow T M \backslash\{0\}$. A regular curve is called a geodesic of spray $S$ if $S \circ c^{\prime}=c^{\prime \prime}$. Locally, $c(t)=\left(x^{i}(t)\right)$ is a geodesic of spray $S$ if

$$
\frac{d^{2} x^{i}}{d t^{2}}+2 G^{i}\left(x, \frac{d x}{d t}\right)=0 .
$$

Definition 3.2. A nonlinear connection (or a horizontal distribution, or Ehresmann connection) is defined by an $n$-dimensional distribution $H: u \in T M \backslash\{0\} \rightarrow H_{u} \subset T_{u}(T M \backslash\{0\})$ that is supplementary to the vertical distribution.

Every spray induces a nonlinear connection through the corresponding horizontal and vertical projectors, [14]

$$
h=\frac{1}{2}\left(\operatorname{Id}-\mathcal{L}_{S} J\right), \quad v=\frac{1}{2}\left(\operatorname{Id}+\mathcal{L}_{S} J\right) .
$$

Locally, the two projectors $h$ and $v$ can be expressed as follows

$$
h=\frac{\delta}{\delta x^{i}} \otimes d x^{i}, \quad v=\frac{\partial}{\partial y^{i}} \otimes \delta y^{i},
$$

where

$$
\frac{\delta}{\delta x^{i}}=\frac{\partial}{\partial x^{i}}-N_{i}^{j}(x, y) \frac{\partial}{\partial y^{j}}, \quad \delta y^{i}=d y^{i}+N_{j}^{i}(x, y) d x^{j}, \quad N_{j}^{i}(x, y)=\frac{\partial G^{i}}{\partial y^{j}}(x, y) .
$$

Horizontal projector $h$ is a vector valued almost semi-basic 1-form.

For a spray $S$ consider the vector valued semi-basic 1 -form

$$
\Phi=-v \circ \mathcal{L}_{S} v=v \circ \mathcal{L}_{S} h=v \circ \mathcal{L}_{S} \circ h,
$$

which will be called the Jacobi endomorphism. It is also known as the Douglas tensor [15, Definition 3.17] or as the Riemann curvature [29, Definition 8.1.2]. Locally, the Jacobi endomorphism can be expressed as follows

$$
\Phi=R_{j}^{i}(x, y) \frac{\partial}{\partial y^{i}} \otimes d x^{j}, \quad R_{j}^{i}=2 \frac{\delta G^{i}}{\delta x^{j}}-S\left(N_{j}^{i}\right)+N_{k}^{i} N_{j}^{k} .
$$

Another important geometric structure induced by a spray $S$ is the curvature tensor $R$. It is the vector valued semi-basic 2 -form

$$
R=\frac{1}{2}[h, h]=\frac{1}{2} R_{j k}^{i} \frac{\partial}{\partial y^{i}} \otimes d x^{j} \wedge d x^{k} .
$$

Locally, the components of the curvature tensor, $R_{j k}^{i}$, are given by

$$
R_{j k}^{i}=\frac{\delta N_{j}^{i}}{\delta x^{k}}-\frac{\delta N_{k}^{i}}{\delta x^{j}}
$$

Curvature tensor $R$ expresses the obstruction to the integrability of the nonlinear connection. Using formulae (2.4) and (3.1) we have that $d_{h}^{2}=d_{R}$.

All the geometric objects induced by a spray $S$ inherit the homogeneity condition. Therefore $[\mathbb{C}, h]=0$, which means that the nonlinear connection is 1 -homogeneous. Also $[\mathbb{C}, R]=0$, $[\mathbb{C}, \Phi]=\Phi$ and hence the the curvature tensor $R$ is 1-homogeneous, while the Jacobi endomorphism $\Phi$ is 2 -homogeneous. 
Using the Jacobi identity, [15, Proposition 2.7] , for the vector valued 0-form $S$ and the vector valued 1 -form $J$ we have $[J,[S, J]]-[J,[J, S]]-[S,[J, J]]=0$. Therefore, we obtain $[J, h]=-2[J,[S, J]]=0$.

The two semi-basic vector vector valued 1 and 2 -forms $\Phi$ and $R$ are related as follows:

$$
\Phi=i_{S} R, \quad[J, \Phi]=3 R
$$

First formula in (3.2) is a consequence of the homogeneity, while the second one is true in a more general context. Locally, the above two formulae can be expressed as follows:

$$
R_{j}^{i}=R_{k j}^{i} y^{k}, \quad R_{j k}^{i}=\frac{1}{3}\left(\frac{\partial R_{k}^{i}}{\partial y^{j}}-\frac{\partial R_{j}^{i}}{\partial y^{k}}\right) .
$$

An important class of sprays, which we will use in the last section to provide examples of projectively metrizable sprays, is that of isotropic sprays, [15, Definition 3.29].

Definition 3.3. A spray $S$ is called isotropic if its Jacobi endomorphism has the form

$$
\Phi=\lambda J+\eta \otimes \mathbb{C}
$$

where $\lambda \in C^{\infty}(T M \backslash\{0\})$ and $\eta$ is a semi-basic 1-form on $T M \backslash\{0\}$.

Due to first formula in (3.2) we have that $i_{S} \Phi=0$ and hence $\lambda=-i_{S} \eta$. Also formulae (3.2) allows us to express the isotropy condition (3.3) for a spray in terms of the curvature tensor $R$.

Proposition 3.4. A spray $S$ is isotropic if and only if its curvature tensor $R$ has the form

$$
R=\alpha \wedge J+\beta \otimes \mathbb{C}
$$

where $\alpha$ is a semi-basic 1 -form and $\beta$ is a semi-basic 2 -form on $T M \backslash\{0\}$.

Proof. We will prove that formulae (3.3) and (3.4) are equivalent.

Suppose that spray $S$ is isotropic. Therefore, the Jacobi endomorphism $\Phi$ satisfies formula (3.3). Using second formula (3.2), the formulae for the Frölicher-Nijenhuis bracket of two vector valued forms $[15$, Appendix A1], and $[J, \mathbb{C}]=J$, we have

$$
3 R=[J, \Phi]=[J, \lambda J+\eta \otimes \mathbb{C}]=\left(d_{J} \lambda-\eta\right) \wedge J+d_{J} \eta \otimes \mathbb{C} .
$$

Hence, the curvature tensor $R$ has the form (3.4).

We assume now that the curvature tensor $R$ has the form (3.4). Using first formula (3.2) and the fact that the inner product $i_{S}$ is a derivation of degree -1 , we have that the Jacobi endomorphism has the form

$$
\Phi=i_{S} R=i_{S} \alpha J+\left(i_{S} \beta-\alpha\right) \otimes \mathbb{C}
$$

Hence, the spray $S$ is isotropic.

We will use Proposition 3.4 and formula (3.4) in Subsection 5.1 to show that isotropic sprays are projectively metrizable sprays. 


\subsection{Projectively related sprays}

Two sprays are projectively equivalent if their geodesics coincide as oriented curves. Therefore, a spray is called projectively metrizable if its geodesics coincide, as oriented curves, with the geodesics of a Finsler space.

In [5] it has been shown that the Helmholtz conditions for an arbitrary semispray to be a Lagrangian vector field can be reformulated in terms of semi-basic 1-forms. It has been shown also that out of the four classic Helmholtz conditions only two of them are necessary and sufficient in the case of the projective metrizability problem for a spray. In this subsection we obtain directly the two Helmholtz conditions, for projective metrizability, in terms of semi-basic 1-forms

Definition 3.5. By a Finsler function we mean a continuous function $F: T M \rightarrow \mathbb{R}$ satisfying the following conditions:

i) $F$ is smooth on $T M \backslash\{0\}$;

ii) $F$ is positive on $T M \backslash\{0\}$ and $F(x, 0)=0$;

iii) $F$ is positively homogeneous of order 1 , which means that $F(x, \lambda y)=\lambda F(x, y)$, for all $\lambda>0$ and $(x, y) \in T M$

iv) the metric tensor with components

$$
g_{i j}(x, y)=\frac{1}{2} \frac{\partial^{2} F^{2}}{\partial y^{i} \partial y^{j}}
$$

has rank $n$.

According to Lovas [21], conditions ii) and iv) of Definition 3.5 imply that the metric tensor $g_{i j}$ of a Finsler function is positive definite.

The regularity condition iv) of Definition 3.5 implies that the Euler-Poincaré 2-form of $F^{2}$, $\omega_{F^{2}}=d d_{J} F^{2}$, is non-degenerate and hence it is a symplectic structure [20, 25]. Therefore, the equation

$$
i_{S} d d_{J} F^{2}=-d F^{2}
$$

uniquely determine a vector field $S$ on $T M \backslash\{0\}$ that is called the geodesic spray of the Finsler function. Equation (3.5) is equivalent to

$$
\mathcal{L}_{S} d_{J} F^{2}=d F^{2} .
$$

Locally, the Euler-Poincaré 2 -form of $F^{2}, \omega_{F^{2}}=d d_{J} F^{2}$, can be expressed as follows

$$
\omega_{F^{2}}=2 g_{i j} \delta y^{i} \wedge d x^{j} .
$$

Definition 3.6. A spray $S$ is called Finsler metrizable if there exists a Finsler function $F$ that satisfies one of the two equivalent conditions (3.5) or (3.6).

One can reformulate condition iv) of Definition 3.5 in terms of the Hessian of the Finsler function $F$ as follows. Consider

$$
h_{i j}(x, y)=F \frac{\partial^{2} F}{\partial y^{i} \partial y^{j}}
$$

the angular metric of the Finsler function. The metric tensor $g_{i j}$ and the angular tensor $h_{i j}$ are related by

$$
g_{i j}=h_{i j}+\frac{\partial F}{\partial y^{i}} \frac{\partial F}{\partial y^{j}} .
$$

Metric tensor $g_{i j}$ has rank $n$ if and only if angular tensor $h_{i j}$ has rank $(n-1)$, see [23]. Therefore, the regularity of the Finsler function $F$ is equivalent with the fact that the Euler-Poincaré 2-form $\omega_{F}=d d_{J} F$ has rank $2 n-2$. 


\section{Definition 3.7.}

i) Two sprays $S_{1}$ and $S_{2}$ are projectively equivalent if their geodesics coincide up to an orientation preserving reparameterization.

ii) A spray $S$ is projectively metrizable if it is projectively equivalent to the geodesic spray of a Finsler function.

Two sprays $S_{1}$ and $S_{2}$ are projectively equivalent if and only if there exists a 1-homogeneous function $P \in C^{\infty}(T M \backslash\{0\})$ such that $S_{2}=S_{1}-2 P \mathbb{C}$, [3, 29].

Next theorem gives a characterization of projectively metrizable sprays in terms of semi-basic 1-forms on $T M \backslash\{0\}$.

Theorem 3.8. A spray $S$ is projectively metrizable if and only if there exists a semi-basic 1-form $\theta \in \Lambda_{v}^{1}(T M \backslash\{0\})$ such that

$$
\begin{aligned}
& \operatorname{rank}(d \theta)=2 n-2, \quad i_{S} \theta>0, \\
& \mathcal{L}_{\mathbb{C}} \theta=0, \quad d_{J} \theta=0, \quad d_{h} \theta=0 .
\end{aligned}
$$

Proof. We prove first that conditions (3.7) and (3.8) are necessary for the projective metrizability problem of the spray $S$. We assume that $S$ is projectively metrizable. Therefore, there exists a Finsler function $F$ with geodesic spray $S_{F}$ and a 1-homogeneous function $P$ on $T M \backslash\{0\}$ such that $S=S_{F}-2 P \mathbb{C}$. Consider $\theta=d_{J} F$, the Euler-Poincaré 1 -form of the Finsler function $F$. Due to the 1 -homogeneity condition of $F$ it follows that $i_{S} \theta=\mathbb{C}(F)=F>0$. The non-degeneracy of the Finsler function implies $\operatorname{rank}(d \theta)=2 n-2$. Since $\theta$ is 0 -homogeneous it follows that $\mathcal{L}_{\mathbb{C}} \theta=0$. Condition $d_{J} \theta=0$ is also satisfied since $d_{J} \theta=d_{J}^{2} F=0$.

It remains to show that $d_{h} \theta=0$. The geodesic spray $S_{F}$ is uniquely determined by condition (3.5), from which it follows that $S_{F}\left(F^{2}\right)=0$ and hence $S_{F}(F)=0$. Since $S_{F}$ also satisfies condition (3.6) it follows that $\mathcal{L}_{S_{F}}(F \theta)=F d F$, which implies $\mathcal{L}_{S_{F}} \theta=d F$. Using $S=S_{F}-2 P \mathbb{C}$ we obtain that $\mathcal{L}_{S} \theta-2 \mathcal{L}_{P \mathbb{C}} \theta=d F$. Using again the 0 -homogeneity of the semi-basic 1-form $\theta$ it follows $\mathcal{L}_{P \mathbb{C}} \theta=P \mathcal{L}_{\mathbb{C}} \theta=0$ and hence $\mathcal{L}_{S} \theta=d F$. We apply now $d_{J}$ to both sides of this last relation and use the commutation rules $\mathcal{L}_{S} d_{J}-d_{J} \mathcal{L}_{S}=d_{[S, J]}=-d_{h}+d_{v}$ and $d d_{J}+d_{J} d=0$. Therefore,

$$
-d_{h} \theta-d_{v} \theta=-d d_{J} F=d_{J} d F=d_{J} \mathcal{L}_{S} \theta=\mathcal{L}_{S} d_{J} \theta+d_{h} \theta-d_{v} \theta
$$

from where it follows that $d_{h} \theta=0$.

We prove now that the conditions (3.7) and (3.8) are sufficient for the projective metrizability problem of the spray $S$. Consider $\theta \in \Lambda^{1}(T M \backslash\{0\})$ a semi-basic 1-form that satisfies conditions (3.7) and (3.8). Define the function $F=i_{S} \theta$. Using the commutation rule $i_{S} d_{J}+d_{J} i_{S}=\mathcal{L}_{\mathbb{C}}-i_{[S, J]}$ as well as conditions $d_{J} \theta=0$ and $\mathcal{L}_{\mathbb{C}} \theta=0$ it follows that $d_{J} F=d_{J} i_{S} \theta=i_{h} \theta=\theta$. Hence $\theta$ is the Euler-Poincaré 1 -form of $F$. Now conditions (3.7) assure that $F$ is a Finsler function. Consider the function $P \in C^{\infty}(T M \backslash\{0\})$ given by $2 P=S(F) / F$, which is 1-homogeneous. We will show now that the spray $\tilde{S}=S-2 P \mathbb{C}$ satisfies equation (3.6) and hence it is the geodesic spray of the Finsler function $F$.

Using the commutation rule $i_{S} d_{h}+d_{h} i_{S}=\mathcal{L}_{S}-i_{[S, h]}$ and the fact that $d_{h} \theta=0$ it follows $0=i_{S} d_{h} \theta=-d_{h} i_{S} \theta+\mathcal{L}_{S} \theta-i_{[S, h]} \theta$. Using the fact that $i_{[S, h]} \theta=d F \circ J \circ \mathcal{L}_{S} h=d F \circ v=d_{v} F$ it follows that $\mathcal{L}_{S} \theta=d_{h} F+d_{v} F=d F$. We show now that $\tilde{S}$ satisfies the same equation. Indeed $\mathcal{L}_{\tilde{S}} \theta=\mathcal{L}_{S-2 P \mathbb{C}} \theta=d F$ since $\mathcal{L}_{P \mathbb{C}} \theta=0$. From the defining formula of function $P$ is follows that $\tilde{S}(F)=S(F)-2 P \mathbb{C}(F)=S(F)-2 P F=0$. Therefore $\mathcal{L}_{\tilde{S}} d_{J} F^{2}=2 F \mathcal{L}_{\tilde{S}} d_{J} F=2 F d F=d F^{2}$ and hence $\tilde{S}$ is the geodesic spray of the Finsler function $F$. 
The second part of the proof of Theorem 3.8 shows that if there exists a semi-basic 1-form $\theta$ on $T M \backslash\{0\}$ that satisfies the conditions (3.7) and (3.8) then the given spray $S$ is projectively related to the spray

$$
S_{F}=S-\frac{\mathcal{L}_{S}\left(i_{S} \theta\right)}{i_{S} \theta} \mathbb{C},
$$

which is the geodesic spray of the Finsler function $F=i_{S} \theta$. In this case, the semi-basic 1-form $\theta=\theta_{i} d x^{i}$ is the Euler-Poincare 1 -form of the Finsler function $F, \theta=d_{J} F$. Therefore,

$$
\theta_{i}=\frac{\partial F}{\partial y^{i}}, \quad h_{i j}=F \frac{\partial \theta_{i}}{\partial y^{j}}, \quad F d \theta=h_{i j} \delta y^{i} \wedge d x^{j} .
$$

Formulae (3.9) show the relation between a semi-basic 1-form $\theta$, a solution of the projective metrizability problem using Theorem 3.8, and the classic approach of the problem using the multiplier matrix $h_{i j}$.

\section{Formal integrability for the projective metrizability problem}

Theorem 3.8 provides necessary and sufficient conditions for the projective metrizability problem. These conditions consist of a set of algebraic equations (3.7), and a set of differential equations (3.8). In this section, we study the set of differential equations (3.8) using Spencer's technique of formal integrability $[7,15]$ and following some of the techniques used for studying the Finsler metrizability problem, which were developed in [26].

\subsection{Formal integrability}

In this subsection, we recall first the basic notions of formal integrability [7, 15] and then we apply it to the system (3.8).

Consider $E$ a vector bundle over the base manifold $M$. For a section $s$ of $E$ and $k \geq 1$ we denote by $j_{x}^{k} s$ the $k$ th order jet of $s$ at the base point $x$ in $M$. The bundle of $k$ th order jets of sections of $E$ is denoted by $J^{k} E$. For two vector bundles $E$ and $F$ over the same base manifold $M$, a linear partial differential operator of order $k$,

$$
P: \operatorname{Sec}(E) \rightarrow \operatorname{Sec}(F),
$$

can be identified with a morphism of vector bundles over $M, p^{0}(P): J^{k} E \rightarrow F$. We will also consider the $l$ th order jet prolongation of the differential operator $P$, which will be identified with the morphisms of vector bundles over $M, p^{l}(P): J^{k+l} E \rightarrow J^{l} F$, defined by

$$
p^{l}(P)\left(j_{x}^{k+l} s\right)=j_{x}^{l}(P s) .
$$

We will denote by $R_{x}^{k+l}(P)=\operatorname{Ker} p_{x}^{l}(P) \subset J_{x}^{k+l} E$ the space of $(k+l)$ th order formal solutions of $P$ at $x$ in $M$.

Definition 4.1. The differential operator $P$ is called formally integrable at $x$ in $M$ if $R^{k+l}(P)$ is a vector bundle over $M$, for all $l \geq 0$, and the map $\bar{\pi}_{x}^{k+l-1}: R_{x}^{k+l}(P) \rightarrow R_{x}^{k+l-1}(P)$ is onto for all $l \geq 1$.

In the analytic case, formal integrability implies existence of analytic solutions for arbitrary initial data, see [7, p. 397].

Denote by $\sigma^{k}(P): S^{k}(M) \otimes E \rightarrow F$ the symbol of $P$, which is defined by the highest order terms of the differential operator $P$, and by $\sigma^{k+l}(P): S^{k+l}(M) \otimes E \rightarrow S^{l}(M) \otimes F$ the symbol of the $l$ th order prolongation of $P$. For each $x$ in $M$, we write

$$
g_{x}^{k}(P)=\operatorname{Ker} \sigma_{x}^{k}(P),
$$




$$
g_{x}^{k}(P)_{e_{1} \ldots e_{j}}=\left\{A \in g_{x}^{k}(P) \mid i_{e_{1}} A=\cdots=i_{e_{j}} A=0\right\}, \quad j \in\{1, \ldots, n\},
$$

where $\left\{e_{1}, \ldots, e_{n}\right\}$ is a basis of $T_{x} M$. Such a basis is called quasi-regular if it satisfies

$$
\operatorname{dim} g_{x}^{k+1}(P)=\operatorname{dim} g_{x}^{k}(P)+\sum_{j=1}^{n} \operatorname{dim} g_{x}^{k}(P)_{e_{1} \ldots e_{j}} .
$$

The symbol $\sigma^{k}(P)$ is called involutive at $x$ in $M$ if there exists a quasi-regular basis of $T_{x} M$.

In this work we will address the projective metrizability problem by discussing first the formal integrability of the system (3.8). For this we will use the two sufficient conditions provided by Cartan-Kähler theorem.

Theorem [Cartan-Kähler]. Let $P$ be a linear partial differential operator of order $k$. Suppose $g^{k+1}(P)$ is a vector bundle over $R^{k}(P)$. If the map $\bar{\pi}^{k}: R^{k+1}(P) \rightarrow R^{k}(P)$ is onto and the symbol $\sigma^{k}(P)$ is involutive, then $P$ is formally integrable.

In order to study the formal integrability of the system (3.8) we consider the first-order partial differential operator $P_{1}: \Lambda_{v}^{1}(T M \backslash\{0\}) \rightarrow \Lambda_{v}^{1}(T M \backslash\{0\}) \oplus \Lambda_{v}^{2}(T M \backslash\{0\}) \oplus \Lambda_{v}^{2}(T M \backslash\{0\})$, which we call the projective metrizability operator

$$
P_{1}=\left(\mathcal{L}_{\mathbb{C}}, d_{J}, d_{h}\right)
$$

Since $\mathbb{C}$ and $J$ are vector valued, semi-basic 0 and respectively 1 -forms and $h$ is a vector valued almost semi-basic 1-form, according to Lemma 2.4, all differential operators $\mathcal{L}_{\mathbb{C}}, d_{J}, d_{h}$ preserve semi-basic forms. Therefore, the differential operator $P_{1}$ is well defined.

\subsection{Involutivity of the projective metrizability operator}

In this subsection we prove that the projective metrizability operator (4.2) satisfies one of the two sufficient conditions for formal integrability, provided by Cartan-Kähler theorem, namely we will prove that the symbol $\sigma^{1}\left(P_{1}\right)$ is involutive.

Since all the bundles we will refer to in this subsection are vector bundles over $T M \backslash\{0\}$, we will omit mentioning it explicitly. For example, we will denote by $T_{v}^{*}$ the vector bundle of semi-basic 1-forms $T_{v}^{*}(T M \backslash\{0\})$, which is a subbundle of $T^{*}(T M \backslash\{0\})$. We will denote by $\Lambda^{k} T_{v}^{*}$ the vector bundle of semi-basic $k$-forms on $T M \backslash\{0\}$, and by $\Lambda_{v}^{k}=\operatorname{Sec}\left(\Lambda^{k} T_{v}^{*}\right)$ the $C^{\infty}(T M \backslash\{0\})$-module of sections $\Lambda_{v}^{k}(T M \backslash\{0\})$. By $S^{k} T^{*}$ we denote the vector bundle of symmetric tensors of $(0, k)$-type on $T M \backslash\{0\}$.

The partial differential operator $P_{1}$ induces a morphism of vector bundles

$$
p^{0}\left(P_{1}\right): J^{1} T_{v}^{*} \rightarrow F_{1}:=T_{v}^{*} \oplus \Lambda^{2} T_{v}^{*} \oplus \Lambda^{2} T_{v}^{*} .
$$

Together with this morphism we will consider the $l$ th order jet prolongations $p^{l}\left(P_{1}\right): J^{l+1} T_{v}^{*} \rightarrow$ $J^{l} F_{1}$, for $l \geq 1$.

Locally, for a semi-basic 1 -form $\theta=\theta_{i} d x^{i} \in \Lambda_{v}^{1}$, we have

$$
\begin{aligned}
\mathcal{L}_{\mathbb{C}} \theta & =\frac{\partial \theta_{i}}{\partial y^{j}} y^{j} d x^{i}, \quad d_{J} \theta=\frac{1}{2}\left(\frac{\partial \theta_{i}}{\partial y^{j}}-\frac{\partial \theta_{j}}{\partial y^{i}}\right) d x^{j} \wedge d x^{i}, \\
d_{h} \theta & =\frac{1}{2}\left(\frac{\delta \theta_{i}}{\delta x^{j}}-\frac{\delta \theta_{j}}{\delta x^{i}}\right) d x^{j} \wedge d x^{i} .
\end{aligned}
$$

Therefore, the vector bundle morphism $p^{0}\left(P_{1}\right)$ can be expressed as follows

$$
p^{0}\left(P_{1}\right)\left(j^{1} \theta\right)=\left(\frac{\partial \theta_{i}}{\partial y^{j}} y^{j} d x^{i}, \frac{1}{2}\left(\frac{\partial \theta_{i}}{\partial y^{j}}-\frac{\partial \theta_{j}}{\partial y^{i}}\right) d x^{j} \wedge d x^{i}, \frac{1}{2}\left(\frac{\delta \theta_{i}}{\delta x^{j}}-\frac{\delta \theta_{j}}{\delta x^{i}}\right) d x^{j} \wedge d x^{i}\right) .
$$


The symbol of $P_{1}$ is the vector bundle morphism $\sigma^{1}\left(P_{1}\right): T^{*} \otimes T_{v}^{*} \rightarrow F_{1}$, defined by the highest order terms of $p^{0}\left(P_{1}\right)$. Since all terms that define $p^{0}\left(P_{1}\right)$ are first-order terms, it follows that

$$
\sigma^{1}\left(P_{1}\right) A=\left(\sigma^{1}\left(\mathcal{L}_{\mathbb{C}}\right) A=i_{\mathbb{C}} A, \sigma^{1}\left(d_{J}\right) A=\tau_{J} A, \sigma^{1}\left(d_{h}\right) A=\tau_{h} A\right) .
$$

In view of formula (2.1), the three components of the vector bundle morphism $\sigma^{1}\left(P_{1}\right)$ are given by:

$$
\begin{aligned}
& \left(\sigma^{1}\left(\mathcal{L}_{\mathbb{C}}\right) A\right)(X)=\left(i_{\mathbb{C}} A\right)(X)=A(\mathbb{C}, X) ; \\
& \left(\sigma^{1}\left(d_{J}\right) A\right)(X, Y)=\left(\tau_{J} A\right)(X, Y)=A(J X, Y)-A(J Y, X) ; \\
& \left(\sigma^{1}\left(d_{h}\right) A\right)(X, Y)=\left(\tau_{h} A\right)(X, Y)=A(h X, Y)-A(h Y, X),
\end{aligned}
$$

for $X, Y$ vector fields on $T M \backslash\{0\}$. Note that for $A \in T^{*} \otimes T_{v}^{*}, i_{\mathbb{C}} A, \tau_{J} A, \tau_{h} A$ are semi-basic forms and hence the symbol $\sigma^{1}\left(P_{1}\right)$ is well defined.

The first-order prolongation of the symbol of $P_{1}$ is the vector bundle morphism $\sigma^{2}\left(P_{1}\right)$ : $S^{2} T^{*} \otimes T_{v}^{*} \rightarrow T^{*} \otimes F_{1}$ that satisfies $i_{X}\left(\sigma^{2}\left(P_{1}\right) B\right)=\sigma^{1}\left(P_{1}\right)\left(i_{X} B\right)$ for all $B \in S^{2} T^{*} \otimes T_{v}^{*}$ and all $X \in \mathfrak{X}(T M \backslash\{0\})$. Therefore, we obtain

$$
\sigma^{2}\left(P_{1}\right) B=\left(\sigma^{2}\left(\mathcal{L}_{\mathbb{C}}\right) B, \sigma^{2}\left(d_{J}\right) B, \sigma^{2}\left(d_{h}\right) B\right),
$$

where for $X, Y, Z$ vector fields on $T M \backslash\{0\}$ we have:

$$
\begin{aligned}
& \left(\sigma^{2}\left(\mathcal{L}_{\mathbb{C}}\right) B\right)(X, Y)=B(X, \mathbb{C}, Y), \\
& \left(\sigma^{2}\left(d_{J}\right) B\right)(X, Y, Z)=B(X, J Y, Z)-B(X, J Z, Y), \\
& \left(\sigma^{2}\left(d_{h}\right) B\right)(X, Y, Z)=B(X, h Y, Z)-B(X, h Z, Y) .
\end{aligned}
$$

Theorem 4.2. The symbol $\sigma^{1}\left(P_{1}\right)$, of the projective metrizability operator $P_{1}=\left(\mathcal{L}_{\mathbb{C}}, d_{J}, d_{h}\right)$, is involutive.

Proof. The symbol $\sigma^{1}\left(P_{1}\right)$ is involutive if there exists a quasi-regular basis of $T_{u}(T M \backslash\{0\})$. It means that we will have to seek for a basis of $T_{u}(T M \backslash\{0\})$ that satisfies the equality (4.1) for $k=1$, at some point $u \in T M \backslash\{0\}$.

We start by computing the first term in the right hand side of formula (4.1), which is $\operatorname{dim} g_{u}^{1}\left(P_{1}\right)$, for some $u \in T M \backslash\{0\}$. Recall that $g^{1}\left(P_{1}\right)=\operatorname{Ker}\left(\sigma^{1}\left(P_{1}\right)\right) \subset T^{*} \otimes T_{v}^{*}$. We have to compute the dimension of the fibers of $g^{1}\left(P_{1}\right)$, which is a vector subbundle of $T^{*} \otimes T_{v}^{*}$. An element $A \in g^{1}\left(P_{1}\right)$ can be expressed, with respect to the adapted dual basis $\left\{d x^{i}, \delta y^{i}\right\}$, as follows

$$
A=A_{i j} d x^{i} \otimes d x^{j}+A_{\underline{i j}} \delta y^{i} \otimes d x^{j}
$$

Using formula (4.3), the symbol $\sigma^{1}\left(P_{1}\right)$ can be expressed as follows:

$$
\sigma^{1}\left(P_{1}\right) A=\left(A_{\underline{i} j} y^{i} d x^{j}, \frac{1}{2}\left(A_{\underline{i} j}-A_{\underline{j} i}\right) d x^{i} \wedge d x^{j}, \frac{1}{2}\left(A_{i j}-A_{j i}\right) d x^{i} \wedge d x^{j}\right) .
$$

The condition $\tau_{h} A=0$ is equivalent with $A_{i j}=A_{j i}$ and due to this condition $A_{i j}$ contribute with $n(n+1) / 2$ to the $\operatorname{dim} g_{u}^{1}\left(P_{1}\right)$. The conditions $\tau_{J} A=0$ and $i_{\mathbb{C}} A=0$ are equivalent to $A_{\underline{i j}}=A_{\underline{j} i}$, and respectively $A_{\underline{i j}} y^{i}=0$. Hence, due to these two conditions, $A_{\underline{i j}}$ contribute with $n(n-1) / 2$ to the the $\operatorname{dim} g_{u}^{1}\left(P_{1}\right)$. It follows that $\operatorname{dim} g_{u}^{1}\left(P_{1}\right)=n(n-1) / 2+n(n+1) / 2=n^{2}$.

We continue the proof by computing the left hand side of formula (4.1), which is $\operatorname{dim} g_{u}^{2}\left(P_{1}\right)$. Therefore, we will consider the kernel of the first-order prolongation of the symbol, $g^{2}\left(P_{1}\right)=$ 
$\operatorname{Ker}\left(\sigma^{2}\left(P_{1}\right)\right) \subset S^{2} T^{*} \otimes T_{v}^{*}$. An element $B \in g^{2}\left(P_{1}\right)$ can be expressed, with respect to the adapted dual basis $\left\{d x^{i}, \delta y^{i}\right\}$, as follows

$$
\begin{aligned}
B= & B_{i j k} d x^{i} \otimes d x^{j} \otimes d x^{k}+B_{\underline{i j k} k} \delta y^{i} \otimes d x^{j} \otimes d x^{k} \\
& +B_{i \underline{j} k} d x^{i} \otimes \delta y^{j} \otimes d x^{k}+B_{i j k} \delta y^{i} \otimes \delta y^{j} \otimes d x^{k},
\end{aligned}
$$

with the symmetry conditions $B_{i j k}=B_{j i k}, B_{\underline{i j k}}=B_{i j k}$ and $B_{i j k}=B_{j i k}$ satisfied. Using formula (4.4), the symbol $\sigma^{2}\left(P_{1}\right)$ can be expressed as follows

$$
\begin{aligned}
\sigma^{2}\left(P_{1}\right) B=( & B_{\underline{i j k}} y^{j} d x^{i} \otimes d x^{k}+B_{\underline{i j k}} y^{j} \delta y^{i} \otimes d x^{k}, \\
& \frac{1}{2}\left(B_{i \underline{j} k}-B_{i \underline{k j}}\right) d x^{i} \otimes d x^{j} \wedge d x^{k}+\frac{1}{2}\left(B_{\underline{i j k}}-B_{\underline{i k j}}\right) \delta y^{i} \otimes d x^{j} \wedge d x^{k}, \\
& \left.\frac{1}{2}\left(B_{i j k}-B_{i k j}\right) d x^{i} \otimes d x^{j} \wedge d x^{k}+\frac{1}{2}\left(B_{\underline{i j k}}-B_{\underline{i k j}}\right) \delta y^{i} \otimes d x^{j} \wedge d x^{k}\right) .
\end{aligned}
$$

The totally symmetric components $B_{i j k}$ contribute with $n(n+1)(n+2) / 6$ to the $\operatorname{dim} g_{u}^{2}\left(P_{1}\right)$. The other two are also totally symmetric components on the $(n-1)$-dimensional space given by restrictions $B_{i \underline{j} k} y^{j}=0$ and respectively $B_{i \underline{j} k} y^{j}=0$. Therefore, each of them contributes with $(n-1) n(n+\overline{1}) / 6$ to the $\operatorname{dim} g_{u}^{2}\left(P_{1}\right)$. Consequently, $\operatorname{dim} g_{u}^{2}\left(P_{1}\right)=n(n+1)(n+2) / 6+2(n-$ 1) $n(n+1) / 6=n^{2}(n+1) / 2$.

Finally, for some $u \in T M \backslash\{0\}$, we seek for a basis of $T_{u}(T M \backslash\{0\})$ for which formula (4.1) holds true. Consider $\left\{h_{i}, i \in\{1, \ldots, n\}\right\}$ a basis for the horizontal distribution and $\left\{v_{i}, i \in\right.$ $\{1, \ldots, n\}\}$, with $v_{n}=\mathbb{C}$, a basis for the vertical distribution such that $J h_{i}=v_{i}$, for all $i \in\{1, \ldots, n\}$.

For $A \in g^{1}\left(P_{1}\right)$, and the basis $\mathcal{B}=\left\{h_{i}, v_{i}, i \in\{1, \ldots, n\}\right\}$, let us denote

$$
a_{i j}=A\left(h_{i}, h_{j}\right) \quad \text { and } \quad b_{i j}=A\left(h_{i}, v_{j}\right) .
$$

It follows that

1. $a_{i j}=a_{j i}, i, j=1, \ldots, n$, because $A \in \operatorname{Ker} \sigma^{1}\left(d_{h}\right)$,

2. $b_{i j}=b_{j i}, i, j=1, \ldots, n$, because $A \in \operatorname{Ker} \sigma^{1}\left(d_{J}\right)$,

3. $b_{n i}=\left(b_{i n}\right)=0, i=1, \ldots, n$, because $A \in \operatorname{Ker} \sigma^{1}\left(\mathcal{L}_{\mathbb{C}}\right)$.

Note that $\operatorname{dim} g^{1}\left(P_{1}\right)=n^{2}$ is determined by the $n(n+1) / 2$ independent components of $a_{i j}$ and $n(n-1) / 2$ independent components of $b_{i j}$. We will prove now that $\widetilde{\mathcal{B}}=\left\{e_{i}, v_{i}, i \in\{1, \ldots, n\}\right\}$, where $e_{1}=h_{1}, e_{2}=h_{2}+v_{1}, \ldots, e_{n-1}=h_{n-1}+v_{n-2}, e_{n}=S+v_{n-1}$, is a quasi-regular basis.

For the basis $\widetilde{\mathcal{B}}$ we denote

$$
\tilde{a}_{i j}=A\left(e_{i}, e_{j}\right) \quad \text { and } \quad \tilde{b}_{i j}=A\left(v_{i}, e_{j}\right) .
$$

Because $A$ is semi-basic in the second variable we have

$$
\begin{aligned}
& A\left(e_{i}, e_{j}\right)=A\left(h_{i}+v_{i-1}, h_{j}+v_{j-1}\right)=A\left(h_{i}+v_{i-1}, h_{j}\right), \\
& A\left(v_{i}, e_{j}\right)=A\left(v_{i}, h_{j}+v_{j-1}\right)=A\left(v_{i}, h_{j}\right),
\end{aligned}
$$

which means that

$$
\tilde{a}_{i j}=a_{i j}+b_{i-1, j}, \quad \tilde{b}_{i j}=b_{i j}, \quad i, j=1, \ldots, n .
$$

Moreover, the $n^{2}$ independent components $a_{i j}$ and $b_{i j}$ of $A$ in the basis $\mathcal{B}$ can be obtained from the components $\tilde{a}_{i j}$ in the basis $\widetilde{\mathcal{B}}$. 
Now, for each $j \in\{1, \ldots, n\}$ we have that conditions $i_{e_{1} \ldots e_{j}} A=0$ give $j n$ independent restrictions on the $n^{2}$-dimensional space $g_{u}^{1}\left(P_{1}\right)$. This implies that

$$
\operatorname{dim} g_{u}^{1}\left(P_{1}\right)_{e_{1} \ldots e_{j}}=n(n-j), \quad \operatorname{dim} g_{u}^{1}\left(P_{1}\right)_{e_{1} \ldots e_{n}, v_{1}, \ldots v_{j}}=0 .
$$

It follows that

$$
\begin{gathered}
\operatorname{dim} g_{u}^{1}\left(P_{1}\right)+\sum_{i=1}^{n} \operatorname{dim} g_{u}^{1}\left(P_{1}\right)_{e_{1}, \ldots, e_{i}}+\sum_{i=1}^{n} \operatorname{dim} g_{u}^{1}\left(P_{1}\right)_{e_{1}, \ldots, e_{n}, v_{1}, \ldots, v_{i}} \\
=n^{2}+n(n-1)+\cdots+n=n^{2}(n+1) / 2=\operatorname{dim} g_{u}^{2}\left(P_{1}\right),
\end{gathered}
$$

which shows that formula $(4.1)$ is satisfied for $P_{1}, k=1$, and the basis $\widetilde{\mathcal{B}}$. Therefore, $\widetilde{\mathcal{B}}$ is a quasi-regular basis and hence the symbol of $P_{1}$ is involutive.

\subsection{First obstruction for the projective metrizability problem}

We have seen in the previous subsection that one condition, of the two sufficient conditions of the Cartan-Kähler theorem, for the formal integrability of $P_{1}$, is satisfied. In this subsection we address the second sufficient condition. We prove that there is only one obstruction for the formal integrability of the projective metrizability operator $P_{1}$ and this is due to the the curvature tensor $R$ of the induced nonlinear connection.

Theorem 4.3. A first-order formal solution $\theta \in \Lambda_{v}^{1}$ of the system (3.8) can be lifted into a second-order solution, which means that $\bar{\pi}_{1}: R^{2}\left(P_{1}\right) \rightarrow R^{1}\left(P_{1}\right)$ is onto, if and only if

$$
d_{R} \theta=0
$$

where $R$ is the curvature tensor (3.1).

Proof. Using the notations from Subsection 4.2, we denote by $K$, the cokernel of the morphism $\sigma^{2}\left(P_{1}\right)$,

$$
K=\frac{T^{*} \otimes\left(T_{v}^{*} \oplus \Lambda^{2} T_{v}^{*} \oplus \Lambda^{2} T_{v}^{*}\right)}{\operatorname{Im} \sigma^{2}\left(P_{1}\right)} .
$$

We will prove the theorem by using the following classical result of homological algebra, see [15, Proposition 1.1]. There exists a morphism $\varphi: R^{1}\left(P_{1}\right) \rightarrow K$ such that the sequence

$$
R^{2}\left(P_{1}\right) \stackrel{\bar{\pi}_{1}}{\longrightarrow} R^{1}\left(P_{1}\right) \stackrel{\varphi}{\longrightarrow} K
$$

is exact. In particular, the morphism $\bar{\pi}_{1}$ is onto if and only if $\varphi=0$.

We will build the morphism $\varphi$ and show that for $\theta \in \Lambda_{v}^{1}$ such that $j_{u}^{1} \theta \in R_{u}^{1}\left(P_{1}\right) \subset J_{u}^{1} T_{v}^{*}$, a first-order solution of $P_{1}$ at $u \in T M \backslash\{0\}$ we have that $\varphi_{u} \theta=0$ if and only if $\left(d_{R} \theta\right)_{u}=0$. The morphism $\varphi$ is represented in the diagram (4.7) by dashed arrows.

To build $\varphi$, we have to define first a morphism of vector bundles

$$
\tau: T^{*} \otimes\left(T_{v}^{*} \oplus \Lambda^{2} T_{v}^{*} \oplus \Lambda^{2} T_{v}^{*}\right) \rightarrow K,
$$


such that the first row in the following diagram is exact.

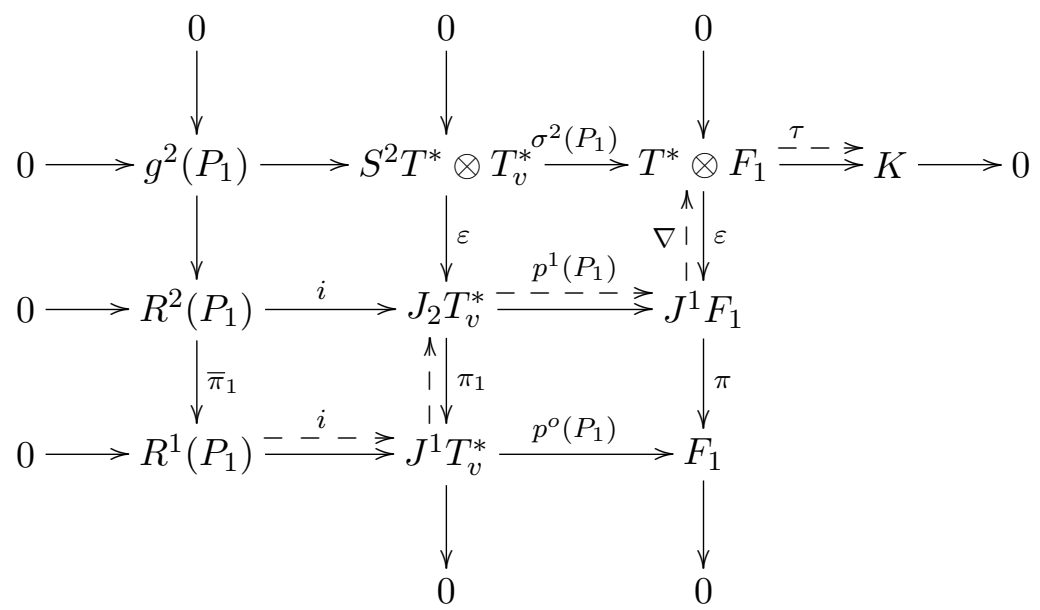

For the vector bundle $K$ given by formula (4.6), the dimension of its fibres is $n^{2}(n-1) / 2$. Therefore, we can view this vector bundle over $T M \backslash\{0\}$ as follows

$$
K=\oplus^{(2)} \Lambda^{2} T_{v}^{*} \oplus^{(3)} \Lambda^{3} T_{v}^{*}
$$

Therefore the map $\tau$ has 5 components. The five components of $\tau=\left(\tau_{1}, \ldots, \tau_{5}\right)$, are given as follows

$$
\begin{array}{ll}
\tau_{1}\left(A, B_{1}, B_{2}\right)=\tau_{J} A-i_{\mathbb{C}} B_{1}, & \tau_{2}\left(A, B_{1}, B_{2}\right)=\tau_{h} A-i_{\mathbb{C}} B_{2}, \\
\tau_{4}\left(A, B_{1}, B_{2}\right)=\tau_{h} B_{2}, & \tau_{5}\left(A, B_{1}, B_{2}\right)=\tau_{h} B_{1}+\tau_{J} B_{2},
\end{array}
$$

for $A \in T^{*} \otimes T_{v}^{*}, B_{1}, B_{2} \in T^{*} \otimes \Lambda^{2} T_{v}^{*}$. Using the above definition of the five components of $\tau$, formula (4.4) that defines the three components of $\sigma^{2}\left(P_{1}\right)$, and the symmetry in the first two arguments of an element $B \in S^{2} T^{*} \otimes T_{v}^{*}$ we can prove $\left(\tau \circ \sigma^{2}\left(P_{1}\right)\right)(B)=0$. For example, the first component of this composition is given by

$$
\begin{aligned}
\left(\tau_{1} \circ \sigma^{2}\left(P_{1}\right)\right)(B)(X, Y) & =\left(\tau_{J} \sigma^{2}\left(\mathcal{L}_{\mathbb{C}}\right)\right)(B)(X, Y)-\left(i_{\mathbb{C}} \sigma^{2}\left(d_{J}\right)\right)(B)(X, Y) \\
& =B(J X, \mathbb{C}, Y)-B(J Y, \mathbb{C}, X)-B(\mathbb{C}, J X, Y)+B(\mathbb{C}, J Y, X)=0 .
\end{aligned}
$$

It follows that $\operatorname{Im} \sigma^{2}\left(P_{1}\right) \subset \operatorname{Ker} \tau$. By comparing the dimensions, it is easy to see that $\operatorname{Im} \sigma^{2}\left(P_{1}\right)=\operatorname{Ker} \tau$, and therefore the first row in diagram (4.7) is exact.

Consider $\nabla$ a linear connection on $T M \backslash\{0\}$ such that $\nabla J=0$. It follows that the connection $\nabla$ preserves the vertical distribution and hence it will preserve semi-basic forms. Therefore, one can view $\nabla$ as a connection on the fibre bundle $F_{1} \rightarrow T M \backslash\{0\}$. Using Lemma 2.5, it follows that derivations $\mathcal{D}_{\mathbb{C}}=i_{\mathbb{C}} \nabla, \mathcal{D}_{J}=\tau_{J} \nabla$, and $\mathcal{D}_{h}=\tau_{h} \nabla$ preserve semi-basic forms. As a firstorder partial differential operator, we can identify connection $\nabla$ with the bundle morphism $p^{0}(\nabla): J^{1} F_{1} \rightarrow T^{*} \otimes F_{1}$. We will use this bundle morphism to define the map $\varphi: R^{1}\left(P_{1}\right) \rightarrow K$ we mentioned at the beginning of the proof.

Consider $\theta \in \Lambda_{v}^{1}$ such that $j_{u}^{1} \theta \in R_{u}^{1}\left(P_{1}\right) \subset J_{u}^{1} T_{v}^{*}$ is a first-order solution of $P_{1}$ at $u \in T M \backslash\{0\}$. Then, we define

$$
\varphi_{u} \theta=\tau_{u} \nabla P_{1} \theta=\tau_{u}\left(\nabla \mathcal{L}_{\mathbb{C}} \theta, \nabla d_{J} \theta, \nabla d_{h} \theta\right) .
$$

We will compute now the five components of map $\varphi$. Since $\mathcal{L}_{\mathbb{C}} \theta, d_{J} \theta$, and $d_{h} \theta$ vanish at $u \in T M \backslash\{0\}$, using Lemma 2.1, it follows that when acting on this semi-basic forms we have 
$\mathcal{D}_{\mathbb{C}}=\mathcal{L}_{\mathbb{C}}, \mathcal{D}_{J}=d_{J}$, and $\mathcal{D}_{h}=d_{h}$. Using the fact that $[J, \mathbb{C}]=J,[h, \mathbb{C}]=0,[J, J]=0$, and $[h, J]=0$, it follows that

$$
\begin{aligned}
\tau_{1}\left(\nabla P_{1} \theta\right)_{u} & =\left(\tau_{J} \nabla \mathcal{L}_{\mathbb{C}} \theta-i_{\mathbb{C}} \nabla d_{J} \theta\right)_{u}=\left(d_{J} \mathcal{L}_{\mathbb{C}} \theta-\mathcal{L}_{\mathbb{C}} d_{J} \theta\right)_{u}=\left(d_{[J, \mathbb{C}]} \theta\right)_{u}=0 ; \\
\tau_{2}\left(\nabla P_{1} \theta\right)_{u} & =\left(\tau_{h} \nabla \mathcal{L}_{\mathbb{C}} \theta-i_{\mathbb{C}} \nabla d_{h} \theta\right)_{u}=\left(d_{h} \mathcal{L}_{\mathbb{C}} \theta-\mathcal{L}_{\mathbb{C}} d_{h} \theta\right)_{u}=\left(d_{[h, \mathbb{C}]} \theta\right)_{u}=0 ; \\
\tau_{3}\left(\nabla P_{1} \theta\right)_{u} & =\left(\tau_{J} \nabla d_{J} \theta\right)_{u}=\left(d_{J}^{2} \theta\right)_{u}=\frac{1}{2}\left(d_{[J, J]} \theta\right)_{u}=0 ; \\
\tau_{4}\left(\nabla P_{1} \theta\right)_{u} & =\left(\tau_{h} \nabla d_{h} \theta\right)_{u}=\left(d_{h}^{2} \theta\right)_{u}=\frac{1}{2}\left(d_{[h, h]} \theta\right)_{u}=\left(d_{R} \theta\right)_{u} ; \\
\tau_{5}\left(\nabla P_{1} \theta\right)_{u} & =\left(\tau_{h} \nabla d_{J} \theta+\tau_{J} \nabla d_{h} \theta\right)_{u}=\left(d_{[h, J]} \theta\right)_{u}=0 .
\end{aligned}
$$

From the above calculations it follows that a first-order formal solution $\theta$ of the system (3.8) can be lifted into a second-order solution if and only if $d_{R} \theta=0$.

Using notation (3.9) we can rewrite obstruction condition (4.5) as an algebraic Bianchi identity for the curvature tensor

$$
F i_{R} d \theta=h_{i k} R_{j l}^{k}+h_{l k} R_{i j}^{k}+h_{j k} R_{l i}^{k}=0 .
$$

An alternative expression for the algebraic Bianchi identity (4.8) was obtained by Szilasi and Vattamány in [31, 4.9.1a].

Using formula (3.1), we obtain that any solution of the system (3.8) necessarily satisfies the curvature obstruction (4.5). In the next section we will discuss various cases when the obstruction (4.5) is automatically satisfied. Another possibility, which we leave for further work, is to add this obstruction to the projective metrizability operator $P_{1}$. In this case we can consider the first-order partial differential operator $P_{2}: \Lambda_{v}^{1}(T M \backslash\{0\}) \rightarrow \Lambda_{v}^{1}(T M \backslash\{0\}) \oplus$ $\Lambda_{v}^{2}(T M \backslash\{0\}) \oplus \Lambda_{v}^{2}(T M \backslash\{0\}) \oplus \Lambda_{v}^{3}(T M \backslash\{0\})$,

$$
P_{2}=\left(\mathcal{L}_{\mathbb{C}}, d_{J}, d_{h}, d_{R}\right)
$$

Following a similar approach as we did for the projective metrizability operator $P_{1}$, we can use the Cartan-Kähler theorem to study the formal integrability of the differential operator $P_{2}$.

\section{Classes of sprays that are projectively metrizable}

In this section we present three classes of sprays for which the projective metrizability operator $P_{1}$ is formally integrable and hence the system (3.8) always has solutions. Therefore, for each of these classes we address the projective metrizability problem, by discussing the set of algebraic conditions (3.7) only, which as we show are always satisfied. We will also provide examples of projectively metrizable sprays that are not Finsler metrizable.

\subsection{Projectively metrizable sprays}

In this subsection we assume that a spray $S$ is analytic, on an analytic manifold $M$. We show that if for spray $S$ the projective metrizability operator $P_{1}$ is formally integrable then the spray is projectively metrizable.

For a semi-basic 1 -form $\theta=\theta_{i}(x, y) d x^{i} \in \Lambda_{v}^{1}$, we will express its first-order jet $j^{1} \theta \in J^{1} T_{v}^{*}$ in the adapted dual basis $\left\{d x^{i}, \delta y^{i}\right\}$, induced by the nonlinear connection associated to the spray, which means

$$
j^{1} \theta=\frac{\delta \theta_{i}}{\delta x^{j}} d x^{j} \otimes d x^{i}+\frac{\partial \theta_{i}}{\partial y^{j}} \delta y^{j} \otimes d x^{i} .
$$


This expression provides us local coordinates $\left(x^{i}, y^{i}, \theta_{i}, \theta_{i j}, \theta_{i j}\right)$ for $J^{1} T_{v}^{*}$. The typical fibre for the fibre bundle $J^{1} T_{v}^{*} \rightarrow T M \backslash\{0\}$ is $\mathbb{R}^{n *} \times L_{2}(n, \mathbb{R}) \times L_{2}(n, \mathbb{R})$. With respect to these local coordinates, the fibre $R_{u}^{1}\left(P_{1}\right)$ of first-order formal solution of $P_{1}$ at $u=\left(x^{i}, y^{i}\right) \in T M \backslash\{0\}$ can be expressed as follows

$$
R_{u}^{1}\left(P_{1}\right)=\left\{\left(x^{i}, y^{i}, \theta_{i}, \theta_{i j}, \theta_{i \underline{j}}\right) \in J_{u}^{1} T_{v}^{*}, \theta_{i j}=\theta_{j i}, \theta_{i \underline{j}}=\theta_{j \underline{i}}, \theta_{i j} y^{j}=0\right\} .
$$

Hence the typical fibre of the fibre bundle $R^{1}\left(P_{1}\right) \rightarrow T M \backslash\{0\}$ is $\mathbb{R}^{n *} \times L_{2, s}(n, \mathbb{R}) \times L_{2, s}(n-1, \mathbb{R})$, where $L_{2, s}(n, \mathbb{R})$ is the space of bilinear symmetric forms on $\mathbb{R}^{n}$.

Consider $\theta$ a solution of the system (3.8), with the initial data $\left(\theta_{i}^{0}, \theta_{i j}^{0}, \theta_{i j}^{0}\right) \in R_{u}^{1}\left(P_{1}\right)$ satisfying the algebraic conditions (3.7). This means $\theta_{i}^{0} y^{i}>0$ (in each fibre, $y^{i}$ is a fixed direction, hence one can choose $\left(\theta_{i}^{0}\right) \in \mathbb{R}^{n *}$ such that $\left.\theta_{i}^{0} y^{i}>0\right)$ and $\operatorname{rank}\left(\theta_{i j}^{0}\right)=n-1\left(\operatorname{choose}\left(\theta_{i j}^{0}\right) \in L_{2, s}(n-1, \mathbb{R})\right.$ and extend it to $\mathbb{R}^{n-1} \oplus\left\{a\left(y^{i}\right), a \in \mathbb{R}\right\}$ such that $\left.\theta_{i j}^{0} y^{j}=0\right)$. If we assume that $M$ is connected and $\operatorname{dim} M \geq 2$, then $T M \backslash\{0\}$ is also connected. Therefore, due to continuity, the solution $\theta$ satisfies the algebraic conditions (3.7), on the connected component of $u \in T M \backslash\{0\}$ where $\theta$ is defined.

We present now some classes of sprays for which the projective metrizability operator $P_{1}$ is always integrable, and hence these sprays will be projectively metrizable. These classes of sprays are:

i) flat sprays, $R=0$;

ii) isotropic sprays, $R=\alpha \wedge J+\beta \otimes \mathbb{C}$, for $\alpha$ a semi-basic 1-form and $\beta$ a semi-basic 2 -form on $T M \backslash\{0\}$;

iii) arbitrary sprays on 2-dimensional manifolds.

For each of these classes of sprays, we will show that the curvature obstruction is automatically satisfied and hence the projective metrizability problem will always have a solution in the analytic case.

In the flat case, the obstruction is automatically satisfied. The fact that flat sprays are projectively metrizable was already demonstrated with other methods in [9].

Assume that a spray $S$ is isotropic. It follows that the curvature tensor has the form $R=$ $\alpha \wedge J+\beta \otimes \mathbb{C}$, for $\alpha \in \Lambda_{v}^{1}$ and $\beta \in \Lambda_{v}^{2}$. Then, for a semi-basic 1-form $\theta$ on $T M \backslash\{0\}$, we have

$$
d_{R} \theta=\alpha \wedge d_{J} \theta+\beta \otimes \mathcal{L}_{\mathbb{C}} \theta
$$

If $\theta$ is a solution of the differential system (3.8) it follows that $\mathcal{L}_{\mathbb{C}} \theta=0$ and $d_{J} \theta=0$, and using formula (5.1) it follows that $d_{R} \theta=0$. Therefore, the obstruction for the formal integrability of $P_{1}$ is satisfied. In [10] it has been shown that any isotropic sprays is projectively equivalent to a flat spray and hence it is projectively metrizable.

If $\operatorname{dim} M=2$ then for a semi-basic 1 -form $\theta$ on $T M \backslash\{0\}, d_{R} \theta$ is a semi-basic 3 -form and hence it will have to vanish. It has been shown by Matsumoto [22] that every spray on a surface is projectively related to a Finsler space, using the original discussion of Darboux [12] about second-order differential equations.

\subsection{Examples}

In this subsection we provide examples of non-metrizable Finsler sprays in the last two of the above mentioned classes of projectively metrizable sprays.

Consider the following system of second-order ordinary differential equations in some open domain in $\mathbb{R}^{2}$, which was proposed by Anderson and Thompson in [2, Example 7.2]:

$$
\frac{d^{2} x^{1}}{d t^{2}}+\left(\frac{d x^{1}}{d t}\right)^{2}+\left(\frac{d x^{2}}{d t}\right)^{2}=0, \quad \frac{d^{2} x^{2}}{d t^{2}}+4 \frac{d x^{1}}{d t} \frac{d x^{2}}{d t}=0 .
$$


The corresponding spray is

$$
S=y^{1} \frac{\partial}{\partial x^{1}}+y^{2} \frac{\partial}{\partial x^{2}}-\left(\left(y^{1}\right)^{2}+\left(y^{2}\right)^{2}\right) \frac{\partial}{\partial y^{1}}-4 y^{1} y^{2} \frac{\partial}{\partial y^{2}} .
$$

It has been shown in [2] that the system (5.2) is not variational and therefore the corresponding spray $S$ in formula (5.3) is not Finsler metrizable. We can also use the techniques from [26] to show that the spray $S$ in formula (5.3) is not Finsler metrizable. However, according to the discussion in the previous subsection, the spray $S$ is projectively metrizable.

Next we consider another example of projectively metrizable spray that is not Finsler metrizable, which was proposed by G. Yang in [32]. Consider $F$ a projectively flat Finsler function on some open domain $U \subset \mathbb{R}^{n}[29, \S 13.5]$. This means that the geodesic spray $S$ of $F$ is projectively equivalent to a flat spray. Therefore, spray $S$ is locally given by:

$$
S=y^{i} \frac{\partial}{\partial x^{i}}-2 P(x, y) y^{i} \frac{\partial}{\partial y^{i}},
$$

where $P$ is 1-homogeneous function on $U \times\left(\mathbb{R}^{n} \backslash\{0\}\right)$. We assume that for the projectively flat Finsler function $F$, its flag curvature is constant $\kappa \in \mathbb{R}, \kappa \neq 0,[15, \S 3.5],[29, \S 11.1]$. This is equivalent to the fact that the Jacobi endomorphism induced by the spray $S$ has the form

$$
\Phi=\kappa\left(F^{2} J-F d_{J} F \otimes \mathbb{C}\right) .
$$

Yang shows in [32, Theorem 1.2] that the projective metrizability class of $S$ contains sprays that are not Finsler metrizable. More precisely, he shows that for $\lambda \in \mathbb{R}$ such that $\lambda \neq 0$ and $\kappa+\lambda^{2} \neq 0$, then the spray

$$
\widetilde{S}=S-2 \lambda F \mathbb{C}
$$

cannot be projectively flat and hence it is not Finsler metrizable.

For spray $\widetilde{S}$ one can compute the corresponding geometric structures: nonlinear connection, Jacobi endomorphism, curvature tensor in terms of the corresponding ones induced by spray $S$ :

$$
\widetilde{h}=h+[P \mathbb{C}, J], \quad \widetilde{\Phi}=\Phi+\lambda^{2}\left(F^{2} J-F d_{J} F \otimes \mathbb{C}\right), \quad \widetilde{R}=R+\lambda^{2} F d_{J} F \wedge J .
$$

Therefore $\widetilde{S}$ has constant flag curvature $\kappa+\lambda^{2}$ and it is also isotropic. Then one can also use formulae (5.5) and Theorem 2 from [26], or Theorem 7.2 from [15], to show that Yang's example given in formula (5.4) is not Finsler metrizable. Yang's example can be extended and it can be shown that for an arbitrary spray, its projective class contains sprays that are not Finsler metrizable, [6].

Therefore, spray $\widetilde{S}$ in formula (5.4) is projectively metrizable but it is not Finsler metrizable.

\section{Acknowledgements}

The work of IB was supported by the Romanian National Authority for Scientific Research, CNCS UEFISCDI, project number PN-II-RU-TE-2011-3-0017. The work of Z.M. has been supported by the Hungarian Scientific Research Fund (OTKA) Grant K67617.

\section{References}

[1] Álvarez Paiva J.C., Symplectic geometry and Hilbert's fourth problem, J. Differential Geom. 69 (2005), 353-378.

[2] Anderson I., Thompson G., The inverse problem of the calculus of variations for ordinary differential equations, Mem. Amer. Math. Soc. 98 (1992), no. 473. 
[3] Antonelli P.L., Ingarden R.S., Matsumoto M., The theory of sprays and Finsler spaces with applications in physics and biology, Kluwer Academic Publisher, Dordrecht, 1993.

[4] Bucataru I., Constantinescu O.A., Dahl M.F., A geometric setting for systems of ordinary differential equations, Int. J. Geom. Methods Mod. Phys. 8 (2011), 1291-1327, arXiv:1011.5799.

[5] Bucataru I., Dahl M.F., Semi basic 1-forms and Helmholtz conditions for the inverse problem of the calculus of variations, J. Geom. Mech. 1 (2009), 159-180, arXiv:0903.1169.

[6] Bucataru I., Muzsnay Z., Projective and Finsler metrizability: parameterization-rigidity of the geodesics, arXiv:1108.4628.

[7] Bryant R.L., Chern S.S., Gardner R.B., Goldschmidt H.L., Griffits P.A., Exterior differential systems, Mathematical Sciences Research Institute Publications, Vol. 18, Springer-Verlag, New York, 1991.

[8] Crampin M., On the differential geometry of the Euler-Lagrange equation and the inverse problem of Lagrangian dynamics, J. Phys. A: Math. Gen. 14 (1981), 2567-2575.

[9] Crampin M., On the inverse problem for sprays, Publ. Math. Debrecen 70 (2007), 319-335.

[10] Crampin M., Isotropic and R-flat sprays, Houston J. Math. 33 (2007), 451-459.

[11] Crampin M., Some remarks on the Finslerian version of Hilbert's fourth problem, Houston J. Math. 37 (2011), 369-391.

[12] Darboux G., Leçons sur la theorie des surfaces, III, Gauthier-Villars, Paris, 1894.

[13] Frölicher A., Nijenhuis A., Theory ot vector-valued differential forms. I. Derivations in the graded ring of differential forms, Nederl. Akad. Wet. Proc. Ser. A 59 (1956), 338-359.

[14] Grifone J., Structure presque-tangente et connexions. I, Ann. Inst. Fourier (Grenoble) 22 (1972), $287-334$.

[15] Grifone J., Muzsnay Z., Variational principles for second-order differential equations. Application of the Spencer theory to characterize variational sprays, World Scientific Publishing Co., Inc., River Edge, NJ, 2000.

[16] Hamel G., Über die Geometrien, in denen die Geraden die Kürzesten sind, Math. Ann. 57 (1903), 231-264.

[17] Klein J., Voutier A., Formes extérieures génératrices de sprays, Ann. Inst. Fourier (Grenoble) 18 (1968), 241-260.

[18] Kolár I., Michor P.W., Slovak J., Natural operations in differential geometry, Springer-Verlag, Berlin, 1993.

[19] Krupková O., Prince G.E., Second order ordinary differential equations in jet bundles and the inverse problem of the calculus of variations, in Handbook of Global Analysis, Editors D. Krupka and D.J. Saunders, Elsevier Sci. B.V., Amsterdam, 2007, 837-904.

[20] de León M., Rodrigues P.R., Methods of differential geometry in analytical mechanics, North-Holland Mathematics Studies, Vol. 158, North-Holland Publishing Co., Amsterdam, 1989.

[21] Lovas R.L., A note on Finsler-Minkowski norms, Houston J. Math. 33 (2007), 701-707.

[22] Matsumoto M., Every path space of dimension two is projectively related to a Finsler space, Open Syst. Inf. Dyn. 3 (1995), 291-303.

[23] Matsumoto M., Foundations of Finsler geometry and special Finsler spaces, Kaiseisha Press, Shigaken, 1986.

[24] Miron R., Anastasiei M., The geometry of Lagrange spaces: theory and applications, Fundamental Theories of Physics, Vol. 59, Kluwer Academic Publishers Group, Dordrecht, 1994.

[25] Morandi G., Ferrario C., Lo Vecchio G., Marmo G., Rubano C., The inverse problem in the calculus of variations and the geometry of the tangent bundle, Phys. Rep. 188 (1990), 147-284.

[26] Muzsnay Z., The Euler-Lagrange PDE and Finsler metrizability, Houston J. Math. 32 (2006), 79-98, math.DG/0602383.

[27] Rapcsák A., Die Bestimmung der Grundfunktionen projektiv-ebener metrischer Räume, Publ. Math. Debrecen 9 (1962), 164-167.

[28] Sarlet W., The Helmholtz conditions revisited. A new approach to the inverse problem of Lagrangian dynamics, J. Phys. A: Math. Gen. 15 (1982), 1503-1517.

[29] Shen Z., Differential geometry of spray and Finsler spaces, Kluwer Academic Publishers, Dordrecht, 2001.

[30] Szilasi J., Calculus along the tangent bundle projection and projective metrizability, in Differential Geometry and its Applications, World Sci. Publ., Hackensack, NJ, 2008, 539-558.

[31] Szilasi J., Vattamány S., On the Finsler-metrizabilities of spray manifolds, Period. Math. Hungar. 44 (2002), 81-100.

[32] Yang G., Some classes of sprays in projective spray geometry, Differential Geom. Appl. 29 (2011), 606-614. 